Pacific Journal of Mathematics

TOPOLOGIES COMPATIBLE WITH HOMEOMORPHISM 


\title{
TOPOLOGIES COMPATIBLE WITH HOMEOMORPHISM GROUPS
}

\author{
Peter Fletcher and Pei LiU
}

If $\mathscr{A}$ is a class of open covers of a topological space $(X, \mathscr{T})$, then $(X, \mathscr{T})$ is said to be strongly $\mathscr{A}$-stable provided that for each $\mathscr{C} \in \mathscr{A}$ there is a homeomorphism $h$ mapping $X$ onto $X$, other than the identity homeomorphism, such that for each $C \in \mathscr{C}$, $h(C)=C$. This paper studies strongly $\mathscr{A}$-stable spaces. Although there are compact connected metric spaces that are not even strongly $\mathscr{A}$-stable with respect to the class $\mathscr{A}$ of all finite open covers, there is an extremely weak homogeneity condition that guarantees that a space $(X, \mathscr{T})$ is strongly $\mathscr{A}$-stable with respect to the class $\mathscr{A}$ of all locally finite open covers. If $H(X)$ is the full homeomorphism group of a space $(X, \mathscr{T})$ that is strongly $\mathscr{A}$-stable with respect to the class $\mathscr{A}$ of all finite open covers, then $H(X)$ is nonabelian and there is a nondiscrete Hausdorff topology $\mathscr{T}$ for $H(X)$ such that $(H(X), \mathcal{T})$ is a topological group.

There has been longstanding interest in topologizing groups of homeomorphisms. For example, in [16], B. L. van der Waerden and D. van Dantzig showed that the group of isometries of a locally compact metric space with only finitely many components is a locally compact topological group under a natural topology; and in [1, Theorem 4] R. Arens showed that the full homeomorphism group of a locally compact, locally connected Hausdorff space forms a topological group under the compact open topology. We say that a topology $\tau$ on a group $(G, \circ)$ is compatible with $(G, \circ)$ provided that $(G, \circ, \tau)$ is a nondiscrete Hausdorff topological group. It has been known for some time that there exist nondegenerate Hausdorff spaces whose only homeomorphism is the identity. Such spaces are now called rigid spaces, and there are now several constructions of rigid spaces in the literature which differ from the earlier folklore example of a rigid metric continuum [9] and [13]. These examples show that none of the familiar topological properties on a space $X$ guarantees that the homeomorphism group $H(X)$ admits a compatible topology. On the other hand A. Kertesz and $T$. Szele have shown that every infinite abelian group admits a compatible topology [11, Theorem 1]. The corresponding problem for infinite nonabelian groups is raised in [11] and to our knowledge remains unsolved.

In section two we define a sequence of increasingly restrictive topological properties for a space $X$, each of which is sufficient to 
guarantee that $H(X)$ is an infinite nonabelian group that admits a compatible topology. These properties are of necessity quite different from the properties usually studied in general topology; however they interact with several standard topological properties and are, we believe, of interest in and of themselves.

In the third section, we introduce a method of constructing topologies on the homeomorphism group of an arbitrary topological space. Our construction makes use of quasi-uniformities and may be considered to be a generalization of a technique of J. Dieudonné; for, in the case that $(X, \mathcal{U})$ is a uniform space, our method yields the topology of uniform convergence. The topology constructed always makes $H(X)$ into a topological group; however, there is no assurance that this topology will be nondiscrete unless the given space $\left(X, \mathscr{T}_{U}\right)$ satisfies one of the properties of section two.

Throughout this paper we let $i$ denote the identity element of a homeomorphism group $H(X)$ and use $H^{*}(X)$ to denote $H(X)-\{i\}$. If $\mathscr{C}$ is a cover of a space $X$, then $A_{x}^{\mathscr{C}}=\cap\{C \in \mathscr{C}: x \in C\}$.

\section{Weak Galois spaces and $\Sigma$-stable spaces.}

Definition [6]. A topological space $(X, \mathscr{T})$ is a Galois space if, for each $U \in \mathscr{T}$ and each $p \in U$, there is $h \in H(X)$ such that $h \mid X-$ $U=i \mid X-U$ and $h(p) \neq p$.

Definition. A topological space $(X, \mathscr{T})$ is a weak Galois space if for each nonempty set $U \in \mathscr{T}$, there is $h \in H(X)$ and $p \in U$ such that $h|X-U=i| X-U$ and $h(p) \neq p$.

Proposition 2.1. Let $(X, \mathscr{T})$ be a Hausdorff weak Galois space. Then $H(X)$ is an infinite nonabelain group.

Proof. Since a weak Galois space has no isolated points and since $(X, \mathscr{T})$ is Hausdorff, it is clear that $X$ is an infinite set. Let $p \in$ $X$. Then there is $h_{1} \in H(X)$ and $x_{1} \in X-\{p\}$ such that $h_{1}\left(x_{1}\right) \neq x_{1}$. Since $X-\left\{p, x_{1}\right\}$ is open, there is $h_{2} \in H(X)$ and $x_{2} \in X-$ $\left\{p, x_{1}\right\}$ such that $h_{2}(p)=p, h_{2}\left(x_{1}\right)=x_{1}$ and $h_{2}\left(x_{2}\right) \neq x_{2}$. One proves by induction that $H(x)$ is infinite.

Let $U$ be a nonempty open set. Then there is $h \in H(X)$ and $p \in U$ such that $h|X-U=i| X-U$ and $h(p) \neq p$. There are neighborhoods $K$ of $p$ and $W$ of $h(p)$ such that $K \cap W=\varnothing$, and there is a neighborhood $M$ of $p$ such that $h(M) \subset W$. Let $V=$ $U \cap k \cap M$. Then $p \in V$ and $V \cap h(V)=\varnothing$. There exists $f \in H(X)$ and $y \in h(V)$ such that $f|X-h(V)=i| X-h(V)$ and $f(y) \neq y$. Let $x=h^{-1}(y)$. Then $x \in V$ and $f(x)=x$. Consequently $h \circ f(x)=$ $h(x)=y \neq f(x)=f \circ h(x)$. 
Definition [3]. An open cover $\mathscr{C}$ of a topological space $(X, \mathscr{T})$ is a $Q$-cover provided that for each $x \in X, A_{x}^{\mathscr{E}} \in \mathscr{T}$.

Definition. Let $(X, \mathscr{T})$ be a topological space and let $\mathscr{C}$ be an open cover of $X$. Then $(X, \mathscr{T})$ is (strongly) $\mathscr{C}$-stable provided that there exists $h \in H^{*}(X)$ such that for each $C \in \mathscr{C}, \quad h(C) \subset C$ $(h(C)=C)$. If $\Sigma$ is a collection of open covers of $X$, then $(X, \mathscr{T})$ is (strongly) $\Sigma$-stable if for each $\mathscr{C} \in \Sigma,(X, \mathscr{T})$ is (strongly) $\mathscr{C}$-stable. If $\Sigma$ is the collection of all finite [locally finite, point finite, $Q-$ ] open covers of $(X, \mathscr{T})$, we say that $(X, \mathscr{T})$ is (strongly) finite [locally finite, point finite, $Q$-] stable.

Definition [7]. A collection $\mathscr{C}$ of subsets of a topological space $(X, \mathscr{T})$ is locally finite somewhere if there exists a nonempty open set $U$ of $X$ that intersects only finitely many members of $\mathscr{C}$.

The proof of the following theorem is similar to that of $[4$, Theorem 4].

THEOREM 2.2. Let $\Sigma$ be a collection of open covers of a weak Galois space $(X, \mathscr{T})$ and suppose that for each $\mathscr{C} \in \Sigma, \mathscr{C}$ is locally finite somewhere. Then $(X, \mathscr{T})$ is strongly $\Sigma$-stable.

Proof. Let $\mathscr{C} \in \Sigma$. Since $\mathscr{C}$ is locally finite somewhere, there exists an open set $V$ such that $V$ intersects only finitely many members of $\mathscr{C}$. Let $\mathscr{F}=\{C \in \mathscr{C}: C \cap V \neq \varnothing\}$ and let

$$
K=\{\mathscr{A} \subset \mathscr{F}: V \cap(\cap \mathscr{A}) \neq \varnothing\} .
$$

The members of $K$ are partially ordered by set inclusion, and since $K$ is finite, there exists a maximal member $\mathscr{F}^{\prime} \in K$. Clearly $V \cap\left(\cap \mathscr{F}^{\prime}\right)$ is open. Since $(X, \mathscr{T})$ is a weak Galois space, there exist $h \in H(X)$ and $x \in V \cap\left(\cap \mathscr{F}^{\prime}\right)$ such that $h \mid X-V \cap\left(\cap \mathscr{F}^{\prime}=i \mid X-V \cap\left(\cap \mathscr{F}^{\prime}\right)\right.$ and $h(x) \neq x$. Let $C \in \mathscr{C}$. If $C \notin \mathscr{F}^{\prime}$, then by maximality of $\mathscr{F}^{\prime}, C \cap V \cap$ $\left(\cap \mathscr{F}^{\prime}\right)=\varnothing$. Hence $C \subset X-V \cap\left(\cap \mathscr{F}^{\prime}\right)$ and we have that $h(C)=$ $C$. Let $C \in \mathscr{F}^{\prime}$ and suppose that for some $p \in C, h(p) \neq p$. Since $h\left|X-V \cap\left(\cap \mathscr{F}^{\prime}\right)=i\right| X-V \cap\left(\cap \mathscr{F}^{\prime}\right), h(p) \in V \cap\left(\cap \mathscr{F}^{\prime}\right) \subset C$. Thus $h(C) \subset C$. Evidently

$$
h^{-1}\left|X-V \cap\left(\cap \mathscr{F}^{\prime}\right)=i\right| X-V \cap\left(\cap \mathscr{F}^{\prime}\right)
$$

so that by an argument similar to that given for $h$, we have that for each $C \in \mathscr{C}, h^{-1}(C) \subset C$. 
In the above theorem, if we take $\Sigma$ to be the collection of all locally finite open covers or to be the collection of all point finite open covers, we obtain the following corollaries:

COROLlary 2.3. Every weak Galois space is strongly locally finite stable.

COROLlaRY 2.4. Every weak Galois space that is of second category is strongly point finite stable.

Proof. A topological space is of second category if and only if every point finite open cover is locally finite somewhere [5, Theorem 1].

Definition. A topological space $(X, \mathscr{T})$ is locally compact at a point $p \in X$ if there exists an open set $U$ containing $p$ such that $\bar{U}$ is compact.

THEOREM 2.5. Let $(X, \mathscr{T})$ be a metrizable weak Galois space that is locally compact at some point. Then $(X, \mathscr{T})$ is a strongly $Q$-stable space.

Proof. Let $\mathscr{C}$ be a $Q$-cover and let $\mathscr{K}=\left\{A_{x}^{\mathscr{E}}: x \in X\right\}$. In order to show that $(X, \mathscr{T})$ is $\mathscr{C}$-stable, it suffices to show that $(X, \mathscr{T})$ is $\mathscr{K}$ stable. For if $(X, \mathscr{T})$ is $\mathscr{K}$-stable, then for each $C \in \mathscr{C}$ and each $x \in C$, $h(x) \in h\left(A_{x}^{\mathscr{E}}\right) \subset A_{x}^{\mathscr{G}} \subset C$. We proceed to show that $(X, \mathscr{T})$ is $\mathscr{K}$-stable.

We first claim that there exists $x \in X$ such that

$$
x \notin \overline{\left.\cup \mathscr{A}_{y}^{\mathscr{C}} \mid x \notin A_{y}^{\mathscr{G}}\right\}} .
$$

Assume the contrary. Then for each $x \in X$, there exists a sequence $\left\{s_{x, n}\right\}_{n=1}^{x}$ such that $\left\{s_{x, n}\right\}$ converges to $x$ and such that

$$
\left\{s_{x, n}\right\} \subset \cup\left\{A_{y}^{\mathscr{C}} \mid x \notin A_{y}^{\mathscr{C}}\right\} \text {. }
$$

Consequently for each positive integer $n, x \notin A_{s x, n}^{\mathscr{C}}$.

Let $x_{1}$ be a point at which $(X, \mathscr{T})$ is locally compact and select an open ball $B_{r_{1}}\left(x_{1}\right)$ of radius $r_{1}$ such that $r_{1}<1$ and $\overline{B_{r_{1}}\left(x_{1}\right)}$ is compact. By the previous argument, there exists a sequence $\left\{s_{x_{1}, n}\right\}$ which converges to $s_{1}$ such that for each positive integer $n, x_{1} \notin A_{s_{x_{1}} n}^{\varepsilon_{n}}$. Thus there exists $s_{x_{1}, n} \in B_{r_{1}}\left(x_{1}\right)$ such that $x \notin A_{s_{x}, n}^{\mathscr{G}}$. Let $x_{2}=s_{x_{1, n}}$. Let $B_{r_{2}}\left(x_{2}\right)$ be an open ball about $x_{2}$ of radius $r_{2}$ such that

$$
B_{r_{1}}\left(x_{1}\right) \cap A_{x_{2}}^{\mathscr{\&}} \supset \overline{B_{r_{2}}\left(x_{2}\right)}
$$


and $r<\frac{1}{2}$. By the previous argument, there exists $x_{3} \in B_{r_{2}}\left(x_{2}\right)$ with $x_{2} \notin A_{x_{3}}^{\varepsilon}$. Let $B_{r_{3}}\left(x_{3}\right)$ be an open ball about $x_{3}$ of radius $r_{3}$ such that $B_{r_{2}}\left(x_{2}\right) \cap A_{x_{3}}^{\mathscr{C}_{3}} \supset \overline{B_{r_{3}}\left(x_{3}\right)}$ and $r_{3}<\frac{1}{3}$. Proceeding as above we obtain by induction a sequence $\left\{B_{r_{i}}\left(x_{i}\right)\right\}$ such that

$$
B_{r_{1}}\left(x_{1}\right) \supset B_{r_{1}}\left(x_{1}\right) \cap A_{x_{2}}^{\mathscr{G}} \supset \overline{B_{r_{2}}\left(x_{2}\right)} \supset B_{r_{2}}\left(x_{2}\right) \cap A_{x_{3}}^{\mathscr{G}} \supset \overline{B_{r_{3}}\left(x_{3}\right)} \supset \ldots
$$

and such that for each positive integer $i, x_{i} \notin A_{x_{i+1}}^{\&}$ and $r_{i}<1 / i$.

Since $\overline{B_{r_{1}}\left(x_{1}\right)}$ is compact, there exists a point $a \in \cap_{i=1}^{\infty} \overline{B_{r_{i}}\left(x_{i}\right)}$. It follows that $a \in \cap_{i=1}^{\infty} A_{x_{1}}^{\mathscr{C}}$, so that $A_{a}^{\mathscr{E}} \subset \cap_{i=1}^{\infty} A_{x_{i}}^{\mathscr{C}}$. But $\left\{x_{i}\right\} \cap$ $\left(\cap_{i=1}^{\infty} A_{x_{1}}^{\mathscr{C}}\right)=\varnothing$. Thus $\left\{x_{i}\right\} \cap A_{a}^{\mathscr{E}}=\varnothing$. Since $A_{a}^{\mathscr{C}}$ is open and $\left\{x_{i}\right\}$ converges to $a$, it is impossible that $\left\{x_{i}\right\} \cap A_{a}^{\mathscr{C}}=\varnothing$.

Therefore there exists a point $p \in X$ such that

$$
p \notin \overline{\cup\left\{A_{y}^{\mathscr{E}} \mid p \notin A_{y}^{\mathbb{B}}\right\}} \text {. }
$$

Let $U$ be an open set about $p$ such that $U \cap\left(\cup\left\{A_{y}^{\mathscr{C}} \mid p \notin A_{y}^{\mathscr{C}}\right\}\right)=\varnothing$ and let $V=U \cap A_{p}^{\mathscr{E}}$. Since $(X, \mathscr{T})$ is a weak Galois space there exists $h \in H^{*}(X)$ with $h|X-V=i| X-V$. Let $A_{y}^{\mathscr{C}} \in \mathscr{K}$ and suppose that $p \notin A_{y}^{\mathscr{E}}$. Then $V \cap A_{y}^{\mathscr{E}}=\varnothing$ and so $h\left(A_{y}^{\mathscr{E}}\right)=A_{y}^{\mathscr{E}}$. If $p \in A_{y}^{\mathscr{E}}$, then $V \subset A_{p}^{\mathscr{C}} \subset A_{y}^{\mathscr{b}}$. For each $x \in A_{y}^{\mathscr{G}}$ such that $h(x) \neq x, x \in V$, and therefore $h(x) \in V \subset A_{p}^{\mathscr{E}} \subset A_{y}^{\mathscr{E}}$. Hence $h$ is $\mathscr{K}$-stable. A similar argument shows that $h^{-1}$ is also $\mathscr{K}$-stable.

In the proof of Theorem 2.5 , the hypothesis that $(X, \mathscr{T})$ is locally compact at a point cannot be omitted, for it has been shown in [7] that the space of rationals with usual topology is not a point finite stable space. (The condition that $(X, \mathscr{T})$ is a weak Galois space cannot be omitted either, since there exist rigid compact metric spaces [9]. Clearly such spaces cannot be $Q$-stable spaces.)

The results of Theorems 2.2 and 2.5 may be summarized by the following diagram:

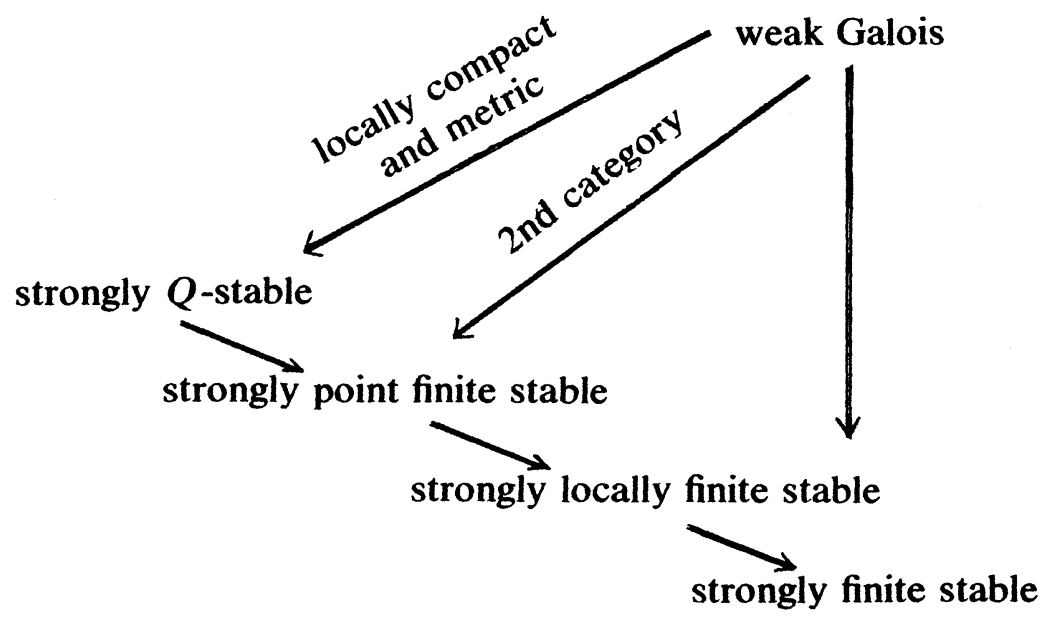




\section{The construction of topologies on homeomorphism} groups.

Definition. Let $X$ be a nonempty set. A quasi-uniformity on $X$ is a filter $U$ or $X \times X$ such that the following hold.

(i) For each $U \in \mathcal{U}, \Delta=\{(x, x): x \in X\} \subset U$.

(ii) For each $U \in \mathcal{U}$, there exists $V \in \mathcal{U}$ with $V \circ V \subset U$.

In this section we assume familiarity with those basic concepts concerning quasi-uniformities that have natural analogues in the study of uniform spaces; these concepts are defined and discussed in detail in [14]. We also make use of the following elementary results.

Let $(X, \mathscr{T})$ be a topological space and let $\Sigma$ be a collection of $Q$-covers of $X$ such that for each $A \in \mathscr{T}$ and each $x \in A$, there exists $\mathscr{C} \in \Sigma$ such that $A_{x}^{\mathscr{C}} \subset A$. For each $\mathscr{C} \in \Sigma$ let

$$
U_{\mathscr{G}}=\cup\left\{\{x\} \times A_{x}^{\mathscr{E}}: x \in X\right\} .
$$

Then $\left\{U_{\mathscr{b}}: \mathscr{C} \in \Sigma\right\}$ is a subbase for a compatible quasi-uniformity $\mathscr{U}_{\Sigma}$ on $(X, \mathcal{T})$.

If $\Sigma$ is the collection of all $Q$ - [point finite, locally finite, finite] open covers of $X$, then $U_{\Sigma}$ is called the fine transitive, [point finite covering, locally finite covering, Pervin] quasi-uniformity. For each $A \in \mathscr{T}$ let $S_{A}=(A \times A) \cup[(X-A) \times X]$. Then the Pervin quasi-uniformity also has a subbase $\left\{S_{A}: A \in \mathscr{T}\right\}$. If $S$ is any subbase for $\mathscr{T}$, then $\left\{S_{A}: A \in\right.$ $S\}$ is a subbase for a compatible quasi-uniformity on $X$ [14].

Definition. Let $(X, \mathcal{U})$ be a quasi-uniform space and for each $U \in \mathcal{U}$ let $W(U)=\{(f, g) \in H(X) \times H(X)$ : for each $x \in X, \quad(f(x)$, $g(x)) \in U\}$. Then $\{W(U): U \in \mathcal{U}\}$ is a base for $W(\mathcal{U})$, the quasiuniformity of quasi-uniform convergence on $H(X)$.

Theorem 3.1 [2, Proposition 4]. Let $(X, \mathcal{U})$ be a quasi-uniform space and let $G$ be a subgroup of $H(X)$ such that each member of $G$ is a U-quasi-uniformly continuous function. Let $G$ have the topology of quasi-uniform convergence with respect to $U$. Then $G$ is a topological semigroup.

The following two examples show that under the hypotheses of Theorem 3.1, $H(X)$ need not be a topological group. We omit the verification of the second example, which, though tedious, is straightforward.

EXAMPLE 3.2. A $T_{0}$ weak Galois space $(X, \mathscr{T})$ which is not $T_{1}$ whose homeomorphism group under the topology of quasi-uniform 
convergence with respect to the Pervin quasi-uniformity forms a topological semigroup that is not a topological group.

Construction. Let $X$ be the set of all real numbers and let the topology on $X$ be

$$
\mathscr{T}=\{(a, b) \subset X:(a, b) \text { is an open interval containing } 0\} \cup\{\varnothing, X\} .
$$

Then clearly $(X, \mathscr{T})$ is a $T_{0}$ space which is not $T_{1}$. Let $h$ be a homeomorphism of $E^{1}$ with the usual topology such that $h(0)=0$. For each nonempty $G \in \mathscr{T}, h^{-1}(G)$ is an open interval containing 0 . Hence $h \in H(X)$. It now follows easily that $(X, \mathscr{T})$ is a weak Galois space.

For each $G \in \mathcal{T}$ let $S_{G}=G \times G \cup(X-G) \times X$. We recall that $S=\left\{S_{G}: G \in \mathscr{T}\right\}$ is a subbase for the Pervin quasi-uniformity $\mathscr{U}$ on $(X, \mathscr{T})$. Since every $h \in H(X)$ is $U$-quasi-uniformly continuous, by the previous theorem, $H(X)$ forms a topological semigroup under the topology of quasi-uniform convergence with respect to $U$.

Define $f:(X, \mathscr{T}) \rightarrow(X, \mathscr{T})$ by $f(x)=-x$. Since $f \in H\left(E^{\prime}\right)$ and $f(0)=0, f \in H(X)$. Clearly $f^{-1}=f$. We claim that the inverse map $\varnothing: H(X) \rightarrow H(X)$ defined by $\varnothing(h)=h^{-1}$ is not continuous at f. Consider the neighborhood $W\left(S_{(-1,1)}\right) f^{-1}$ and let $W\left(S_{(a . b)}\right) f$ be a basic neighborhood of $f$ in $H(X)$. Consider the function $g:(X, \mathscr{T}) \rightarrow(X, \mathscr{T})$ defined by $g(x)=-\frac{1}{2} x$. Then $g \in H(X)$. Moreover we now show that $g \in W\left(S_{(a, b)}\right) f$. Let $x \in X$.

$$
\begin{aligned}
& \text { If }-x \in(a, b), \text { then } a<\frac{a}{2}<\frac{-x}{2}<\frac{b}{2}<b . \text { Thus }(f(x), g(x))= \\
& \left(-x, \frac{-x}{2}\right) \in(a, b) \times(a, b) \subset S_{(a, b) .} \\
& \text { If }-x \notin(a, b) \text {, then } \\
& (f(x), g(x))=\left(-x, \frac{-x}{2}\right) \in(X-(a, b)) \times X \subset S_{(a, b) .} .
\end{aligned}
$$

Therefore $g \in W\left(S_{(a . b)}\right) f$. We note that $g^{-1}(x)=-2 x$ and that $g^{-1} \notin W\left(S_{(-1.1)} f^{-1}\right.$, since

$$
\begin{aligned}
&\left(f^{-1}(3 / 4), g^{-1}(3 / 4)\right)=\left(\frac{-3}{4}, \frac{-3}{2}\right) \notin(-1,1) \times(-1,1) \\
& \cup(X-(-1,1)) \times X=S_{(-1,1)} .
\end{aligned}
$$

EXAMPLE 3.3. A quasi-uniformity $U$ compatible with the usual topology on the closed interval $I=[-1,1]$ which has the property that every $h \in H(I)$ is U-quasi-uniformly continuous and $H(I)$ is not a topological group under the topology of quasi-uniform convergence with respect to $\mathcal{U}$. 
Construction. Let $U$ be the quasi-uniformity on $I$ having subbase $S=\left\{S_{G}: G=(a, b)\right.$ or $(a, 1]$ or $\left.[-1, b)\right\}$ where $-1<a<b<1$. It may be seen that the inverse map is not continuous at $-i$.

Definition. A collection $\Sigma$ of $Q$-covers of a topological space $X$ is a topological collection provided that if $\mathscr{C} \in \Sigma$, then for all $h \in H(X)$, $\{h(C): C \in \mathscr{C}\} \in \Sigma$.

Lemma. Let $(X, \mathscr{T})$ be a topological space and let $\Sigma$ be a topological collection of $Q$-covers such that if $x \in A \in \mathscr{T}$, there is $\mathscr{C} \in \Sigma$ with $A_{x}^{\mathscr{C}} \subset A$. Then $H(X)$ is a 0-dimensional topological group under the topology of uniform convergence with respect to the uniformity $u_{\Sigma} \vee \mathcal{U}_{\Sigma}^{-1}$.

Proof. We note that every $h \in H(X)$ is $U_{\Sigma}$-quasi-uniformly continuous so that every $h \in H(X)$ is $\mathscr{U}_{\Sigma} \vee \mathcal{U}_{\Sigma}^{-1}$-uniformly continuous. Thus $H(X)$ is a topological group [2, Proposition 4]. Since for each $\mathscr{C} \in \Sigma, U_{\mathscr{C}} \circ U_{\mathscr{C}}=U_{\mathscr{C}}, U_{\Sigma} \vee U_{\Sigma}^{-1}$ has a base of equivalence relations. Thus $\mathscr{T}_{W\left(U_{\Sigma \vee} \cup \bar{\Sigma}_{\Sigma}^{\prime}\right)}$ is 0 -dimensional.

THEOREM 3.4. Let $(X, \mathscr{T})$ be a topological space and let $\Sigma$ be the collection of all $Q$ - [point finite, locally finite, finite open] covers of $(X, \mathscr{T})$. Let $(X, \mathscr{T})$ be strongly $\Sigma$-stable. Then $H(X)$ is a nondiscrete 0 -dimensional topological group under the topology of uniform convergence with respect to $\mathcal{U}_{\Sigma} \vee \mathcal{U}_{\Sigma}^{-1}$.

Proof. Since one easily sees that $\Sigma$ is a topological collection of $Q$-covers, by the preceding lemma it suffices to show that $\mathscr{T}_{W}\left(\mathcal{U}_{\Sigma} \vee \mathcal{U}_{\Sigma}^{-1}\right)$ is nondiscrete.

Let $G$ be an open set about $i$. Then there exist basic members $K$ and $V$ of $U_{\Sigma}$ such that $W\left(K \cap V^{-1}\right) i \subset G$. Let $\mathscr{A}_{j}, j=1,2, \cdots, n$, and $\mathscr{B}_{j}, j=1,2, \cdots, m$ be members of $\Sigma$ such that $K=\cap_{j=1}^{n} U_{\mathscr{A}_{1}}$ and $V=\cap{ }_{j=1}^{m} U_{\mathscr{B}_{j}}$ and let $\mathscr{C}_{1}=\cup_{j=1}^{n} \mathscr{A}_{j}, \mathscr{C}_{2}=\cup_{j=1}^{m} \mathscr{B}_{j}$. Then $\mathscr{C}_{1}$ and $\mathscr{C}_{2}$ are members of $\Sigma$. We have that $K=\bigcap_{j=1}^{n} U_{\mathscr{A}_{j}}=U_{\cap_{j=1}^{n} \mathscr{A}_{j}}=U_{\mathscr{C}_{1}}$ and that $V=\cap{ }_{j=1}^{m} U_{\mathscr{B}_{1}}=U_{\cup_{j=1}^{m} \mathscr{B}_{1}}=U_{\mathscr{C}_{2}}$. Since $(X, \mathscr{T})$ is strongly $\Sigma$-stable, there exists $h \in H^{*}(X)$ such that both $h$ and $h^{-1}$ are $\mathscr{C}_{1} \cup \mathscr{C}_{2}$-stable.

Since $h$ is $\mathscr{C}_{1}$-stable, $h$ is $\left\{A_{x}^{\mathscr{C}_{1}}: x \in X\right\}$-stable. For each $x \in X$, $h(x) \subset A_{x}^{\mathscr{C}_{1}}$. Therefore for each $x \in X,(x, h(x)) \in\{x\} \times A_{x}^{\mathscr{C}_{1}} \subset U_{\mathscr{C}_{1}}=$ $K$. Hence $h \in W(K) i$. Let $x \in X$ and let $y=h(x)$. Then $x \in A_{y}^{\mathscr{C}_{2}}$. Otherwise $h^{-1}(y)=x \notin A_{y}^{\mathscr{C}_{2}}$ whereas $h^{-1}$ is $\mathscr{C}_{2^{-}}$ stable. Therefore $(h(x), x) \in\{y\} \times A_{y}^{\mathscr{C}_{2}} \subset U_{\mathscr{C}_{2}}=V \quad$ and $\quad(x, h(x))$ $\in V^{-1}$. Hence $h \in W\left(V^{-1}\right) i$. Finally we have that 


$$
h \in W(K) i \cap W\left(V^{-1}\right) i=\left[W(K) \cap W\left(V^{-1}\right)\right] i=W\left(K \cap V^{-1}\right) i \subset G .
$$

Thus $H(X)$ is a nondiscrete topological group.

Corollary. Let $(X, \mathscr{T})$ be a $T_{0}$ weak Galois space. Then $H(X)$ admits a compatible topology $\mathscr{T}^{\prime}$ such that $\left(H(X), \mathscr{T}^{\prime}\right)$ is a Galois space.

Proof. Let $\mathcal{U}$ be the locally finite covering quasi-uniformity for $(X, \mathscr{T})$. Then by Corollary $2,3,(X, \mathscr{T})$ is strongly locally finite stable. Thus $H(X)$ is a nondiscrete topological group under the topology of uniform convergence with respect to $U \vee U^{-1}$. Since $(X, \mathscr{T})$ is $T_{0}, H(X)$ is a $T_{0}$ space with respect to the compact open

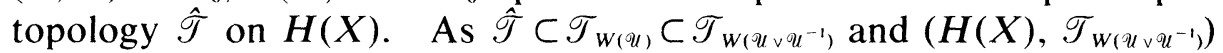
is a topological group, $\mathscr{T}_{W\left(u \sim u^{-1}\right)}$ is Hausdorff. By Theorem $3.4, H(X)$ is 0 -dimensional and homogeneous so that $H(X)$ is a Galois space [7, Page 529].

Corollary. Let $(X, \mathscr{T})$ be a second countable $T_{0}$ weak Galois space. Then $H(X)$ admits a compatible metric topology.

Proof. Let $\mathscr{B}$ be a countable base for $\mathscr{T}$ and let $\mathcal{U}$ be the quasi-uniformity on $(X, \mathscr{T})$ having subbasis

$$
S=\{(G \times G) \cup[(X-G) \times X]: G \in \mathscr{B}\} .
$$

Then $\mathcal{U}$ has a countable base and so $\mathcal{U} \vee \mathcal{U}^{-1}$ also has a countable base. Let $\mathscr{L}$ be the locally finite covering quasi-uniformity for $(X, \mathscr{T})$. Since $U \subset \mathscr{L}$, the topology of uniform convergence on $H(X)$ with respect to $\mathcal{U} \vee \mathcal{U}^{-1}$ is nondiscrete. The argument that $\mathscr{T}_{W\left(U_{\vee} \mathcal{U}^{-1}\right)}$, is Hausdorff is the same as that of the previous corollary.

If $(X, \mathscr{T})$ is a weak Galois completely regular Hausdorff space, then the topology of uniform convergence with respect to the fine uniformity is compatible with $H(X)$ [12]. This result and the first corollary above motivate the following questions:

Is every infinite nonabelian group isomorphic to the full homeomorphism group of a $T_{0}$ weak Galois space? If so, can the $T_{0}$ weak Galois space also be taken to be completely regular?

The result of [8] that every group is isomorphic to the full homeomorphism group of a complete, connected, locally connected metric space gives some reason to hope that the questions raised above can be answered affirmatively. Evidently, however, the techniques employed in [8] are not applicable here. 


\section{REFERENCES}

1. R. Arens, Topologies for homeomorphism groups, Amer. J. Math., 68 (1946), 593-610.

2. J. Dieudonné, On topological groups of homeomorphisms, Amer. J. Math., 70 (1948), 659-680.

3. P. Fletcher, On completeness of quasi-uniform spaces, Arch. Math. (Basel) 22 (1971), 200-204.

4. P. Fletcher, Homeomorphism groups with the topology of quasi-uniform convergence, Arch. Math. (Basel), 22 (1971), 88-93.

5. P. Fletcher and W. F. Lindgren, A note on spaces of second category, Arch. Math. (Basel), 24 (1973), 186-187.

6. P. Fletcher and R. L. Snider, Topological Galois spaces, Fund. Math., 68 (1970), 143-148.

7. J. R. Fogelgren and R. A. McCoy, Some topological properties defined by homeomorphism groups, Arch. Math. (Basel), 22 (1971), 528-533.

8. J. de Groot, Groups represented by homeomorphism groups, I, Math. Annalen, 138 (1959), 80-102.

9. J. de Groot and R. J. Wille, Rigid continua and topological group pictures, Arch. Math. (Basel), 9 (1958), 441-446.

10. J. de Groot and R. H. McDowell, Autohomeomorphism groups of 0-dimensional spaces, Compositio Math., 15 (1963), 203-209.

11. A. Kertesz and T. Szele, On existence of non-discrete topologies in infinite abelian groups, Publ. Math. Debrecen, 3 (1953), 187-189.

12. Pei Liu, Nondiscrete topologies on homeomorphism groups of uniform spaces, submitted for publication.

13. F. W. Lozier, A class of compact rigid 0-dimensional spaces. Canad. J. Math., 21 (1969), 817-821.

14. M. G. Murdeshwar and S. A. Naimpally, Quasi-uniform Topological Spaces, Noordhoff (1966).

15. W. J. Pervin, Quasi-uniformization of topological spaces, Math. Ann., 147 (1962), 316-317.

16. B. L. van der Waerden and D. van Dantzig. Über metrisch homogene Räume, Abhandlungen aus dem Mathematischen Seminar der Hamburgischen Universität, 6 (1928), 367-376.

Received October 16, 1973 and in revised form April 4, 1974.

Virginia Pol ytechnic Institute:

AND

State University 



\section{Pacific Journal of Mathematics}

\section{Vol. 56, No. $1 \quad$ November, 1975}

Shimshon A. Amitsur, Central embeddings in semi-simple rings .......... 1

David Marion Arnold and Charles Estep Murley, Abelian groups, A, such

that $\operatorname{Hom}(A,---)$ preserves direct sums of copies of $A \ldots \ldots \ldots .$.

Martin Bartelt, An integral representation for strictly continuous linear

operators ................................... 21

Richard G. Burton, Fractional elements in multiplicative lattices......... 35

James Alan Cochran, Growth estimates for the singular values of

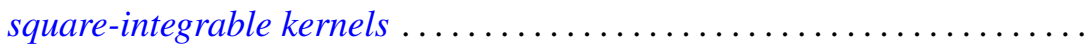

C. Martin Edwards and Peter John Stacey, On group algebras of central

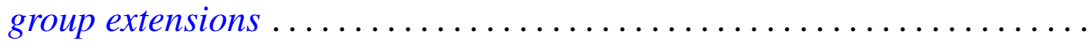

Peter Fletcher and Pei Liu, Topologies compatible with homeomorphism

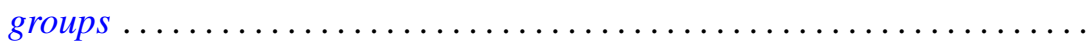

George Gasper, Jr., Products of terminating ${ }_{3} F_{2}(1)$ series ............ 87

Leon Gerber, The orthocentric simplex as an extreme simplex ............

Burrell Washington Helton, A product integral solution of a Riccati

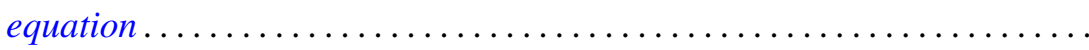

Melvyn W. Jeter, On the extremal elements of the convex cone of

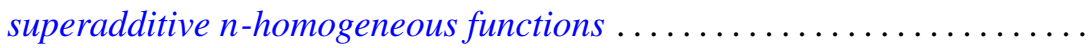

R. H. Johnson, Simple separable graphs .

Margaret Humm Kleinfeld, More on a generalization of commutative and

alternative rings. . .

A. Y. W. Lau, The boundary of a semilattice on an $n$-cell.

Robert F. Lax, The local rigidity of the moduli scheme for curves ...

Glenn Richard Luecke, A note on quasidiagonal and quasitriangular

operators .

Paul Milnes, On the extension of continuous and almost periodic functions

Hidegoro Nakano and Kazumi Nakano, Connector theory.

James Michael Osterburg, Completely outer Galois theory of perfect rings ..................................

Lavon Barry Page, Compact Hankel operators and the F. and M. Riesz

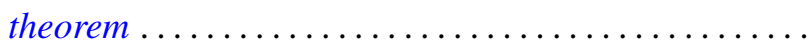

Joseph E. Quinn, Intermediate Riesz spaces..................... 225

Shlomo Vinner, Model-completeness in a first order language with a generalized quantifier.

Jorge Viola-Prioli, On absolutely torsion-free rings ..........

Philip William Walker, A note on differential equations with all solutions of integrable-square............................

Stephen Jeffrey Willson, Equivariant maps between representation 